Estudo Reológico da Pectina Extraída da Casca de Maracujás

\author{
Nascimento Filho, W. B.; Melo Filho, A. A.
}

Rev. Virtual Quim., 2016, 8 (3), 838-855. Data de publicação na Web: 17 de maio de 2016

http://rvq.sbq.org.br

\title{
Rheological Study of Pectin Extracted from the Passion Fruit Bark
}

Abstract: Pectin has been used as dietary fiber to have beneficial effects on the human body. Although the albedo citrus are the most common sources of commercial pectin extraction, pectins have different characteristics such as degree of esterification, particle size, rheological, solubility and gelling ability, which is directly connected to the raw material ultilizada for their extraction which results in the need to study alternative extraction sources. The aim of this study was to determine some viscometric parameters (kinematic viscosity, intrinsic viscosity, constant viscosimetric and average molecular weight viscosimetric by graphical extrapolation methods and the determination from a single point) of pectin extracted from three species of passion fruit (Passiflora edulis, alata and Passiflora quadrangular) grown in the state of Roraima. The diluted solutions of pectin showed an exponential behavior as a function of increasing temperature, characterizing them as Newtonian fluids. The coefficient of determination obtained by exponential regression of kinematic viscosity data of pectin solutions were (0.9), which is a great fit for the viscosity curves. It was determined by graphical extrapolation intrinsic viscosity method (Huggins; Kraemer, Martin; Schulz-Blaschke) and a single point (Schulz-Blaschke; Solomon and Ciuta; and Deb Chanterjee). It was found that the method of determining by a single point is not suitable for obtaining intrinsic viscosity for this system. Negative values $\mathrm{KH}$ demonstrated good polymer solvation for the pectin chains. It determined the mean square distance between the ends of the polymer chains by means of Flory's equation, using the viscometric data (Huggins), and the molecular weight obtained by the equation of Mark Houwink-Sakurada, obtaining values in a range of $5.44 \times 10^{-22} 4.26 \times 10^{-23} \mathrm{~cm}$.

Keywords: Viscometry; Viscosimetric molecular weight; Viscosity; Intrinsic viscosity.

\section{Resumo}

A pectina vem sendo utilizada como fibra dietética por apresentar efeitos benéficos ao organismo humano. Embora os albedos cítricos sejam as fontes mais usuais para extração de pectina comercial, as pectinas apresentam características diferentes, como grau de esterificação, tamanho das partículas, propriedades reológicas, solubilidade e capacidade de gelificação, que estar diretamente ligado a matéria-prima ultilizada para sua extração o que acarreta na necessidade do estudo de fontes alternativas de extração. $O$ objetivo deste trabalho foi determinar alguns parâmetros viscosimétricos (viscosidade cinemática, viscosidade intrínseca, constantes viscosimétricas e massa média molecular viscosimétrica pelos métodos de extrapolação gráfica e pela determinação a partir de um único ponto) da pectina extraída de três espécies de maracujás (Passiflora edulis, Passiflora alata e Passiflora quadrangulares) cultivadas no estado de Roraima. As soluções diluídas de pectina apresentaram um comportamento exponencial em função do aumento da temperatura, caracterizando-as como fluidos newtonianos. O coeficiente de determinação obtido através da regressão exponencial dos dados de viscosidade cinemática das soluções de pectina foram $(0,9)$, sendo este um ótimo ajuste para as curvas de viscosidade. Determinou-se a viscosidade intrínseca pelo método de extrapolação gráfica (Huggins; Kraemer; Martin; Schulz-Blaschke) e por um único ponto (Schulz-Blaschke; Solomon e Ciuta; e Deb e Chanterjee). Verificou-se que o método de determinação por um único ponto não foi apropriado para obtenção da viscosidade intrínseca para este sistema. Os valores negativos de $\mathrm{K}_{\mathrm{H}}$ demonstraram uma boa solvatação para as cadeias poliméricas de pectina. Determinou-se a distância média quadrática entre as extremidades das cadeias poliméricas por meio da equação de Flory, utilizando-se os dados viscosimétricos (Huggins), bem como as massas moleculares obtidas por meio da equação de Mark HouwinkSakurada, obtendo-se valores em uma faixa de $5,44 \times 10^{-22}$ a $4,26 \times 10^{-23} \mathrm{~cm}$.

Palavras-chave: Viscosimetria; Massa molecular viscosimétrica; Viscosidade cinemática; Viscosidade intrínseca.

* Universidade Federal de Roraima, Av. Capitão Ene Garcez, 2413, Boa Vista, CEP 69304-000, Departamento de Química, Roraima,
Brasil.
DOI: 10.5935/1984-6835.20160061




\section{Estudo Reológico da Pectina Extraída da Casca de Maracujás Wilson B. do Nascimento Filho, * Antonio A. de Melo Filho}

Universidade Federal de Roraima, Av. Capitão Ene Garcez, 2413, Boa Vista, CEP 69304-000, Departamento de Química, Roraima, Brasil.

*wilson botelho@hotmail.com

Recebido em 18 de setembro de 2015. Aceito para publicação em 16 de maio de 2016

\section{Introdução}

\section{Parte Experimental}

2.1. Obtenção das Amostras e Extração da Pectina

2.2. Determinação da Viscosidade Cinemática

2.3. Determinação da Viscosidade Intrínseca e Massa Molecular Média Viscosimétrica

2.4. Obtenção das Constantes de Huggins, Kraemer, Martin e Schulz-Blasck

2.5. Determinação da Concentração Critica de Overlap

2.6. Determinação do Tamanho das Cadeias Poliméricas

\section{Resultados e Discussão}

\section{Conclusão}

\section{Introdução}

O gênero Passiflora é composto por 465 espécies, das quais, 150 a 200 são originárias do Brasil e cerca de 70 espécies produzem frutos comestíveis. ${ }^{1}$ As espécies Passiflora edulis ( $P$. edulis) e Passiflora Alata (P. Alata) ocorrem em várias formações florestais nas regiões sudeste e sul do Brasil, enquanto que a Passiflora quadrangulares ( $P$. quadrangulares) é endêmica da floresta Amazônica.

O maracujá é uma fruta muito utilizada na indústria alimentícia, cosmética e farmacêutica e, como efeito desta utilização, tem-se a geração de uma grande quantidade de resíduos de cascas e sementes, que podem ser reutilizados para a extração da pectina e óleo, agregando valor a estes subprodutos. $^{2}$

O Brasil é o maior produtor de maracujá do mundo, contribuindo com uma produção de 838.244 toneladas, em 2013, segundo os dados do Instituto Brasileiro de Geografia e Estatística (IBGE), ${ }^{3}$ seguido pela Colômbia, Peru e Equador. ${ }^{4}$ Considerado, portanto, maior exportador e consumidor do mundo, 5,6 segundo dados que se referem à $P$. edulis (maracujá amarelo). A espécie $P$. quadrangularis (maracujá melão) e $P$. alata (maracujá doce) ${ }^{7}$ vêm sendo comercializadas nas feiras e mercados com boa aceitação popular por apresentar alto teor de açúcar, proteínas, vitaminas e sais minerais, podendo 
ser consumidos in natura ou industrializados através de geleias, sucos e sorvetes. ${ }^{8,9}$

O maracujá é utilizado nas indústrias para produção de sucos, gerando toneladas de resíduos provenientes de cascas e sementes que possuem descarte inadequado ou são utilizados para alimentação animal. Deste fruto, cerca de $70 \%$ são rejeitados, como as cascas e as sementes, e $30 \%$ são utilizados na produção de sucos. ${ }^{10,11} \mathrm{Na}$ literatura, constam diversos trabalhos sobre reaproveitamento destes resíduos, explorando o elevado teor de pectina da casca e de óleo das sementes. Assim, toda a fruta seria aproveitada, desde a polpa para a fabricação de sucos e geleia, casca para a produção de pectina até as sementes para a extração do óleo. ${ }^{12}$

A pectina é um dos principais constituintes estruturais da parede celular das plantas dicotiledôneas e de algumas monocotiledôneas, com inúmeras propriedades que permitem usá-la como agente gelificante, espessante e estabilizante. ${ }^{13,14} \mathrm{~A}$ estrutura das moléculas de pectina é composta por polímeros lineares em uma cadeia principal de ácidos D-galacturônico, Figura 1, unidos por ligações glicosídicas $\alpha$ $(1 \rightarrow 4)$, apresentando uma parte na forma de éster metílico. ${ }^{15,16}$

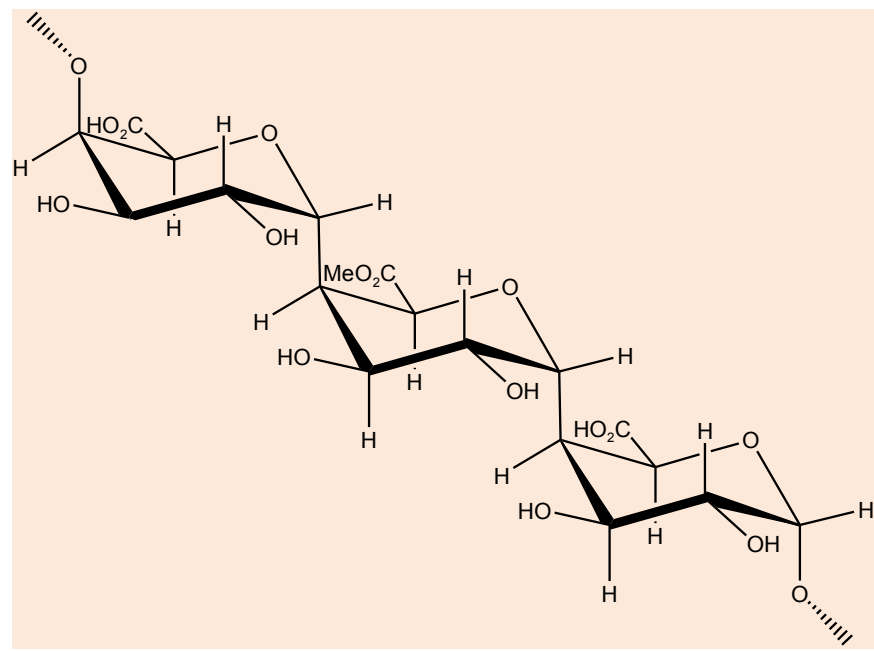

Figura 1. Estrutura química da cadeia de pectina ${ }^{15}$

As cadeias de pectina são interrompidas por unidades de $(1 \rightarrow 2)$ - $\alpha$-L-ramnose, contendo, além disso, uma porção de cadeias ramificadas de açúcares neutros, como Dglicose, L-arabinose, D-galactose e D-xilose. ${ }^{15}$

A importância da pectina em alimentos é geralmente atribuída à formação de géis, que são amplamente usados na produção de gomas, geleias, produtos lácteos, entre outros. ${ }^{17,18}$ Nos últimos anos, a pectina também vem sendo empregada como fibra dietética solúvel por apresentar efeitos fisiológicos benéficos ao organismo humano, tais como redução dos níveis de colesterol, lipoproteínas, ácidos biliares e glicose. ${ }^{19-21}$

As fontes mais usuais para a extração de pectina comercial são os albedos cítricos e o bagaço de maçã. ${ }^{22,23}$ Dependendo da fonte de extração, pectinas apresentam características diferentes, como grau de esterificação, tamanho das partículas, conteúdo de açúcares, teor de cinzas, propriedades reológicas, entre outros, tendo, consequentemente, propriedades funcionais diferentes (solubilidade, capacidade e condição de gelificação), ${ }^{24}$ o que estimula o estudo de fontes alternativas de extração. Atualmente, existem inúmeras pesquisas sobre extração de pectina de diferentes fontes, ressaltando-se o aproveitamento de resíduos de indústrias. ${ }^{18,25}$

O estudo reológico pode ser definido como a área que investiga as deformações e 
o fluxo da matéria quando submetida a uma tensão. ${ }^{26}$ A caracterização reológica é um parâmetro que fornece resultados importantes. Pode-se avaliar, por exemplo, a qualidade de um solvente pelos valores de viscosidade intrínseca $([\eta]),{ }^{23}$ bem como obter a massa molecular de polímeros através da equação de Mark-HouwinkSakurada. Esta equação relaciona a massa molecular viscosimétrica (Mv) do polímero com duas constantes, $K$ e $\alpha$, que por sua vez, estão relacionadas com o polímero e 0 solvente utilizados a uma determinada temperatura. ${ }^{27,28}$

A simplicidade do método de análise e dos equipamentos envolvidos faz a viscosimetria uma técnica de fácil execução em qualquer laboratório, tanto no meio acadêmico, quanto no industrial. $^{23}$

Torna-se essencial o estudo de novas fontes para extração de pectina, além de seu estudo reológico, uma vez que o mesmo é considerado uma técnica de simples execução e que fornece dados associados ao comportamento estrutural do material. Portanto, o objetivo deste trabalho foi determinar alguns parâmetros viscosimétricos (viscosidade cinemática, viscosidade intrínseca, constantes viscosimétricas e massa média molecular viscosimétrica pelos métodos de extrapolação gráfica e pela determinação a partir de um único ponto) da pectina extraída de três espécies de maracujás ( $P$. edulis, $P$. alata e P.quadrangulares) cultivadas no estado de Roraima, uma vez que são incipientes as informações reológicas sobre estas espécies. $^{29}$

\section{Parte Experimental}

\subsection{Obtenção das Amostras e Extração da Pectina}

Os maracujás ( $P$. edulis, $P$. quadrangularis e $P$. alata) foram coletados nos municípios de
Boa Vista e Pacaraima, no estado de Roraima. As amostras foram levadas ao Laboratório de Produtos Naturais do Departamento de Química da Universidade Federal de Roraima, UFRR, onde foram lavadas com água corrente. A pectina foi extraída utilizando uma solução de ácido cítrico $0,086 \%$ e tempo de extração de 60 minutos a $97^{\circ} \mathrm{C}$.

\subsection{Determinação da Viscosidade Cinemática}

As análises foram realizadas em triplicatas, com variação da temperatura de $293,15 \mathrm{~K}$ a 353,15 K. As amostras de pectina foram solubilizadas na concentração de $1 \mathrm{~g} / \mathrm{L}^{-}$ ${ }^{1}$ com solução tampão fosfato de sódio 0,01 $\mathrm{M}, \mathrm{pH}$ 7. Foi utilizado um viscosímetro AVS 350 marca Schott com banho termostatizado CT52 e viscosímetro capilar Cannon-Fenske de $\mathrm{n}^{\circ} 100$ para obtenção do tempo de escoamento das soluções das pectinas dos maracujás e da pectina cítrica industrial. A Equação 1 foi utilizada para determinar a viscosidade cinemática. ${ }^{31,32}$

$$
\eta_{\text {cin }}=K(t-v)
$$

Onde:[?] $]_{\text {cin }}$ Viscosidade Cinemática $\mathrm{mm}^{2} . \mathrm{s}^{-1} ; \mathrm{K}$ : Constante de Capilar $\mathrm{n}^{\circ} 100$; $\mathrm{t}$ : É a média do tempo de escoamento; $v=$ correção $d a$ energia cinética.

A temperatura é um parâmetro relacionado com a energia interna da substância ou mistura. A literatura relata que a viscosidade de um líquido é altamente influenciada por mudanças da temperatura. A viscosidade dos líquidos incompressíveis varia inversamente com a temperatura absoluta, apresentando um comportamento exponencial. ${ }^{33}$ Realizou-se uma análise de regressão exponencial dos dados obtidos na viscosidade cinemática utilizando o software Origin 6.0. 
2.3. Determinação da Viscosidade Intrínseca e Massa Molecular Média Viscosimétrica

As análises foram realizadas a $25{ }^{\circ} \mathrm{C}$. As amostras foram solubilizadas em uma faixa de concentração de $1,0 \mathrm{~g} . \mathrm{L}^{-1}$ a 5,0 g.L $\mathrm{L}^{-1}$. Foram anotadas três medidas de tempo para cada concentração com um cronômetro digital. Foi utilizado um viscosímetro capilar CannonFenske $\mathrm{n}^{\circ} 100$ para a obtenção do tempo de escoamento das soluções de pectina. A viscosidade intrínseca foi determinada com o cálculo do incremento da viscosidade especifica (Equação 2) dividindo esta, pela concentração da solução (Equação 3). . $2,34,35^{-3}$

$$
\eta_{\text {sp }}=\frac{\left(\eta_{\text {cin. da solução }}-\eta_{\text {cin. do solvente }}\right)}{\eta_{\text {cin. do solvente }}}
$$

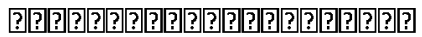

$$
\eta_{\text {red }}=\eta_{\text {sp }} / c
$$

Onde $\eta_{\mathrm{sp}}=$ viscosidade específica; $\eta_{\text {red }}=$ Viscosidade reduzida; $c=$ concentração $d a$ solução.

A viscosidade intrínseca de Huggins é definida como o valor de viscosidade reduzida à diluição infinita $(C \rightarrow 0)$. A viscosidade intrínseca da pectina ([n]) foi determinada através do gráfico da viscosidade reduzida $\left(\eta_{\text {red }}\right)$ versus concentração (C) com extrapolação à concentração zero do polímero (coeficiente linear), ${ }^{28,35,36}$ conforme a Equação 4.

$$
[\eta]_{\mathrm{h}}=\lim _{c \rightarrow 0} \eta_{\text {red }}
$$

As viscosidades intrínsecas de Kraemer, Martin e Schulz-Blascke (Equações 5-7) foram determinadas a fim de comparar os métodos empregados para determinação de parâmetros viscosimétricos. ${ }^{28,35-37}$

$$
\begin{aligned}
& {[\eta]_{k}=\lim _{c \rightarrow 0} \eta_{\text {inh }}} \\
& {[\eta]_{m}=\lim _{c \rightarrow 0} \ln \eta_{\text {red }}} \\
& {[\eta]_{s b}=\lim _{c \rightarrow 0} \eta_{\text {red }}}
\end{aligned}
$$

A massa molecular média viscosimétrica das amostras de pectina foi determinada pela aplicação da equação de Mark HouwinkSakurada, a qual relaciona [ $\eta$ ] com Mv. ${ }^{28,34,38}$

$$
[\eta]=K\left[M_{v}\right]^{\alpha}
$$

Mv: Massa molecular média viscosimétrica; $\mathrm{K}$ é uma constante com valor de 0,30 e $\alpha 0,613$. $^{34}$

2.4. Obtenção das Constantes de Huggins, Kraemer, Martin e Schulz-Blasck

As constates de Huggins $\left(K_{h}\right)$, Kraemer $\left(K_{k}\right)$, Martin $\left(K_{m}\right)$ e Schulz-Blascke $\left(K_{b s}\right)$ foram obtidas através das Equações 9-12. 14,28,35

$$
\begin{aligned}
& \eta_{\text {red }}=[\eta]_{h}+K_{h}[\eta]^{2} c \\
& \ln \eta_{r} / c=[\eta]_{k}-K_{k}[\eta]_{k}^{2} c \\
& \ln \eta_{s p} / c=\ln [\eta]_{m}+K_{m}[\eta]_{m} c \\
& \eta_{s p} / c=[\eta]_{s b}+k_{s b}[\eta]_{s b} \eta_{s b}
\end{aligned}
$$

Onde $\bigoplus_{s p}$ é a viscosidade específica definida pela equação 2; 国 é a viscosidade relativa: $\eta_{r}=\left(\eta_{\text {cin. da solução }} / \eta_{\text {cin. do solvente }}\right)$.

Para determinação da viscosidade intrínseca a partir de um único ponto, algumas relações têm sido propostas. Combinando as equações 9 e 10, Solomon e Ciuta ${ }^{37}$ chegaram a Equação 13 para a determinação da viscosidade intrínseca. 


$$
[\eta]=\left[2\left(\eta_{s p}-\ln \eta_{r}\right)\right]^{1 / 2} / c
$$

A Equação 14 foi proposta por Deb e Chanterjee $^{32}$ como uma expressão da viscosidade intrínseca determinada pelo método de um único ponto. $O$ uso das equações 13 e 14 tem sido feito a partir da suposição que $k_{h}+k_{k}=0,5{ }^{28}$

$$
[\eta]=\left(3 \ln \eta_{r}+3 / 2 \eta_{s p}^{2}-3 \eta_{s p}\right) / c
$$

$k_{h}, k_{k}, k_{m}$, e $k_{s b}=$ constantes de Huggins, Kraemer, Martin e Schulz-Blaschke, respectivamente. Para muitos sistemas poliméricos, o valor de $k_{s b}$ é $0,28 .{ }^{39}$

\subsection{Determinação da Concentração Critica de Overlap}

A concentração crítica de overlap pode ser definida como aquela, a partir da qual, se observa um desvio da linearidade na relação $\log \eta_{\mathrm{sp}} \times \log \mathrm{c}[\eta] .^{28}$

\subsection{Determinação do Tamanho das Cadeias Poliméricas}

As dimensões das cadeias poliméricas podem ser medidas a partir dos dados viscosimétricos por meio da equação de Flory $(15)^{28,40}$

$$
[\eta]=\frac{\phi\left(h^{2}\right)^{3 / 2}}{M_{v}}
$$

Onde $\mathrm{Mv}$ é a massa molecular média viscosimétrica, $\phi$ é uma constante igual a $2,1 \times 10^{21} \mathrm{com}[\eta]$ dada em $100 \mathrm{~mL} \cdot \mathrm{g}^{-1}$ para polímeros lineares e $\left(h^{2}\right)^{1 / 2}$ é raiz quadrada da distância média quadrática extremo-aextremo $(\mathrm{cm})$ da cadeia polimérica.

\section{Resultados e Discussão}

Newton relaciona a viscosidade como um fator determinante no escoamento dos fluidos. Esse fenômeno pode ser descrito pela lei de Newton, que determina que a deformação de um fluido cresce contínua e linearmente, qualquer que seja a tensão aplicada. ${ }^{28}$

A definição newtoniana clássica para a viscosidade é $\eta=$ ? $/$ ? , sendo $\eta$ a viscosidade, [? a tensão de cisalhamento (força/área cisalhada) e $\gamma$ a taxa de deformação ou gradiente de velocidade. Se a viscosidade for independente da tensão aplicada ou do gradiente de velocidade, o fluido é denominado newtoniano. ${ }^{28}$

Fluidos não newtonianos são aqueles que apresentam a característica de variar a viscosidade em função da tensão aplicada ou de seu gradiente de velocidade. Na prática, o comportamento newtoniano está restrito a moléculas de baixa massa molecular. Porém, soluções poliméricas se comportam como fluidos newtonianos em concentrações próximas a zero (solução diluída). ${ }^{28,41} \mathrm{~A}$ Figura 2 apresenta os regimes de concentração de soluções poliméricas a qual o regime newtoniano é caracterizado por $\mathrm{C}<$ C*. 


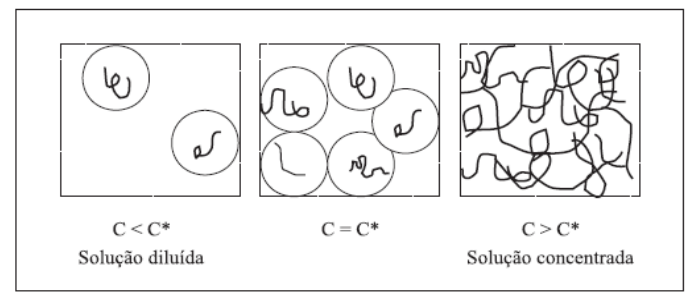

Figura 2. Representação esquemática dos regimes de concentração de uma solução polimérica. Adaptado da referência 23

A formação de entrelaçamentos em um sistema polimérico (polímero-solvente) é derivada da interação molecular e esta é diretamente ligada com o aumento da massa molecular do polímero o que resulta no aumento de sua viscosidade. ${ }^{25}$

A concentração crítica $\left(\mathrm{C}^{*}\right)$ reflete o volume ocupado pela cadeia polimérica isolada. A uma concentração acima desta, observa-se mudanças nas propriedades de escoamento devido aos entrelaçamentos intermoleculares que ocorrem, $\left(\mathrm{C}>\mathrm{C}^{*}\right)$.
Avaliando concentrações mais baixas do que $C^{*}$, observa-se a liberdade no movimento individual dos novelos poliméricos $\left(C<C^{*}\right)$, ou seja, as soluções estão em regime newtoniano. ${ }^{25}$

A Figura 3 apresenta a relação entre os

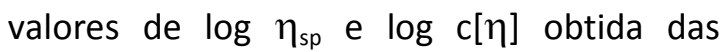
soluções de pectina em solução tampão fosfato de sódio $\mathrm{pH} 7$ a 298,15 K, empregando-se $\mathrm{o}$ valor fornecido pela viscosidade intrínseca de Huggins.

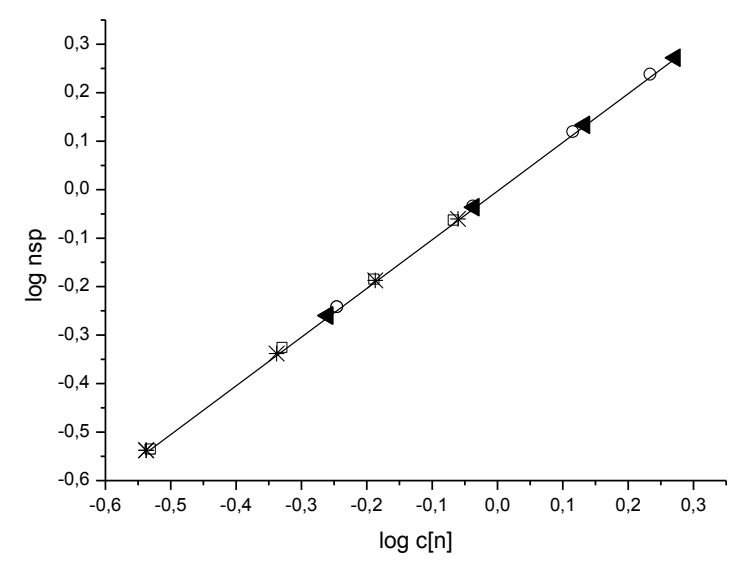

Figura 3. Log nsp versus log c[n] para as soluções de pectinas: (০) Cítrica; ( ४) P. edulis; ( $\square$ ) $P$. alata; $\left({ }^{*}\right)$ P. quadrangularis em solução tampão fosfato de sódio $\mathrm{pH} 7$

A concentração crítica $\left(C^{*}\right)$ pode ser definida como aquela, a partir da qual, se observa um desvio da linearidade na relação. O parâmetro ("dimensionless coil overlap parameter", onde $c$ é a concentração utilizada para determinar a viscosidade intrínseca de Huggins), é definido como o volume total ocupado pelos novelos poliméricos, c[n] é a medida da fração em volume do polímero presente na solução. Esse parâmetro é independente da massa molecular e do tipo de polímero. ${ }^{40,42}$ Foi observada uma relação linear para todas as soluções de pectina analisadas na faixa de concentração de 1,0 g. $\mathrm{L}^{-1}$ a 5,0 g.L $\mathrm{L}^{-1}$, indicando que todas foram realizadas em regime newtoniano. 
Os valores de viscosidade cinemática em função da temperatura das soluções de pectina das espécies de maracujás supracitadas juntamente com a de pectina cítrica industrial estão apresentados na
Tabela 1. A viscosidade cinemática é medida observando-se o tempo que um fluido leva para escoar através dos orifícios de um capilar sob a força da gravidade. ${ }^{43,44}$

Tabela 1. Valores de viscosidade cinemática das soluções de pectina em função do aumento da temperatura

\begin{tabular}{ccccc}
\hline \multicolumn{2}{c}{ Temperatura } & \multicolumn{3}{c}{ Viscosidade Cinemática $\left(\mathrm{mm}^{\left.\mathbf{2} . \mathbf{s}^{-1}\right)}\right.$} \\
\hline Kelvin (K) & P. edulis & P. alata & P. quadrangularis & Pectina cítrica industrial \\
$\mathbf{2 9 3 , 1 5}$ & $1,43 \pm 0,01$ & $1,29 \pm 0,22$ & $1,29 \pm 0,06$ & $1,34 \pm 0,18$ \\
$\mathbf{3 0 3 , 1 5}$ & $1,12 \pm 0,09$ & $1,02 \pm 0,01$ & $1,01 \pm 0,30$ & $1,05 \pm 0,31$ \\
$\mathbf{3 1 3 , 1 5}$ & $0,91 \pm 0,02$ & $0,82 \pm 0,02$ & $0,82 \pm 0,19$ & $0,86 \pm 0,02$ \\
$\mathbf{3 2 3 , 1 5}$ & $0,75 \pm 0,01$ & $0,72 \pm 0,17$ & $0,71 \pm 0,15$ & $0,73 \pm 0,01$ \\
$\mathbf{3 3 3 , 1 5}$ & $0,67 \pm 0,04$ & $0,60 \pm 0,08$ & $0,62 \pm 0,10$ & $0,61 \pm 0,05$ \\
$\mathbf{3 4 3 , 1 5}$ & $0,57 \pm 0,07$ & $0,53 \pm 0,03$ & $0,54 \pm 0,01$ & $0,54 \pm 0,02$ \\
$\mathbf{3 5 3 , 1 5}$ & $0,47 \pm 0,01$ & $0,47 \pm 0,01$ & $0,48 \pm 0,01$ & $0,48 \pm 0,05$ \\
\hline
\end{tabular}

Newton faz uma relação da viscosidade para uma força que determinado fluido adquire ao escoar por uma superfície, observando uma relação linear, sendo a sua constante de proporcionalidade a viscosidade do fluido. Assim, todos os fluidos que seguem este comportamento são denominados fluidos newtonianos. ${ }^{28,44}$ Para Canteri e colaboradores, $^{41}$ a pectina, em soluções diluídas, apresenta comportamento de fluidos newtonianos. A Figura 4 apresenta a viscosidade cinemática das soluções de pectina extraída das cascas dos maracujás estudados e da pectina industrial.

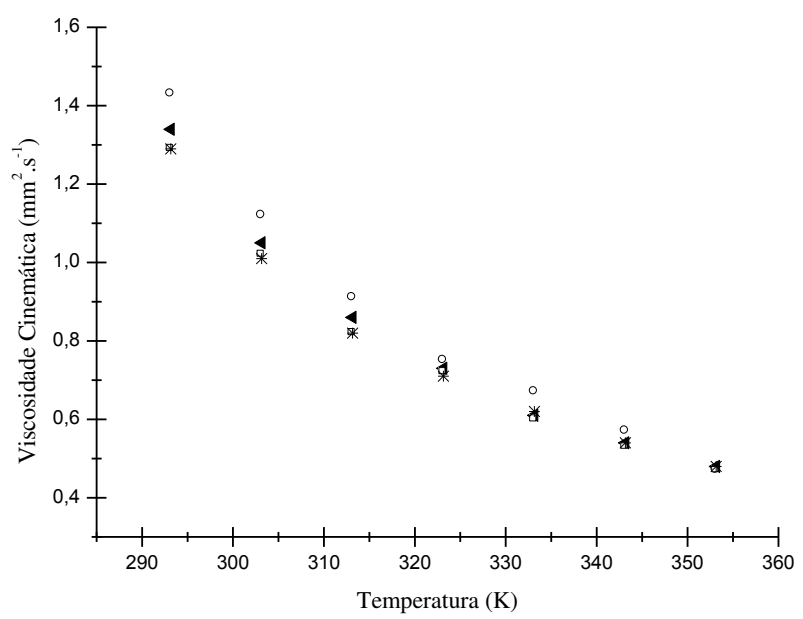

Figura 4. Viscosidade Cinemática $\left(\mathrm{mm}^{2} \cdot \mathrm{s}^{-1}\right)$ das soluções de pectina em função do aumento de temperatura. oPectina Cítrica industrial; $\varangle P$. edulis; $\square P$. alata; * $P$. quadrangularis 
As análises de viscosidade em função da temperatura das soluções de pectina mostraram um comportamento exponencial, Figura 4, com o aumento da temperatura, podendo ser observada uma redução da viscosidade de todas as soluções. Foi realizada uma análise de regressão exponencial, (Figura 5) dos dados obtidos da viscosidade cinemática utilizando o software Origin 6.0.
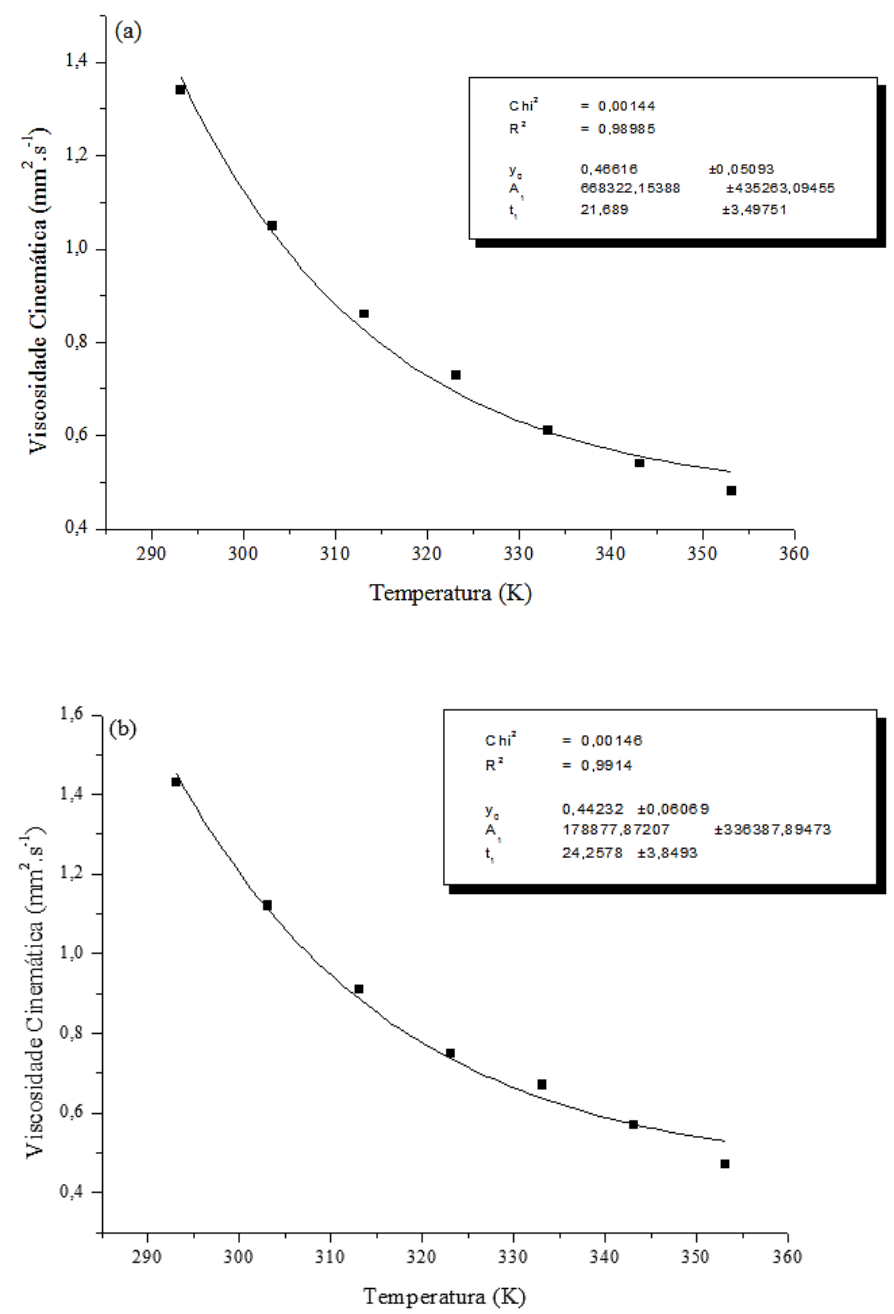

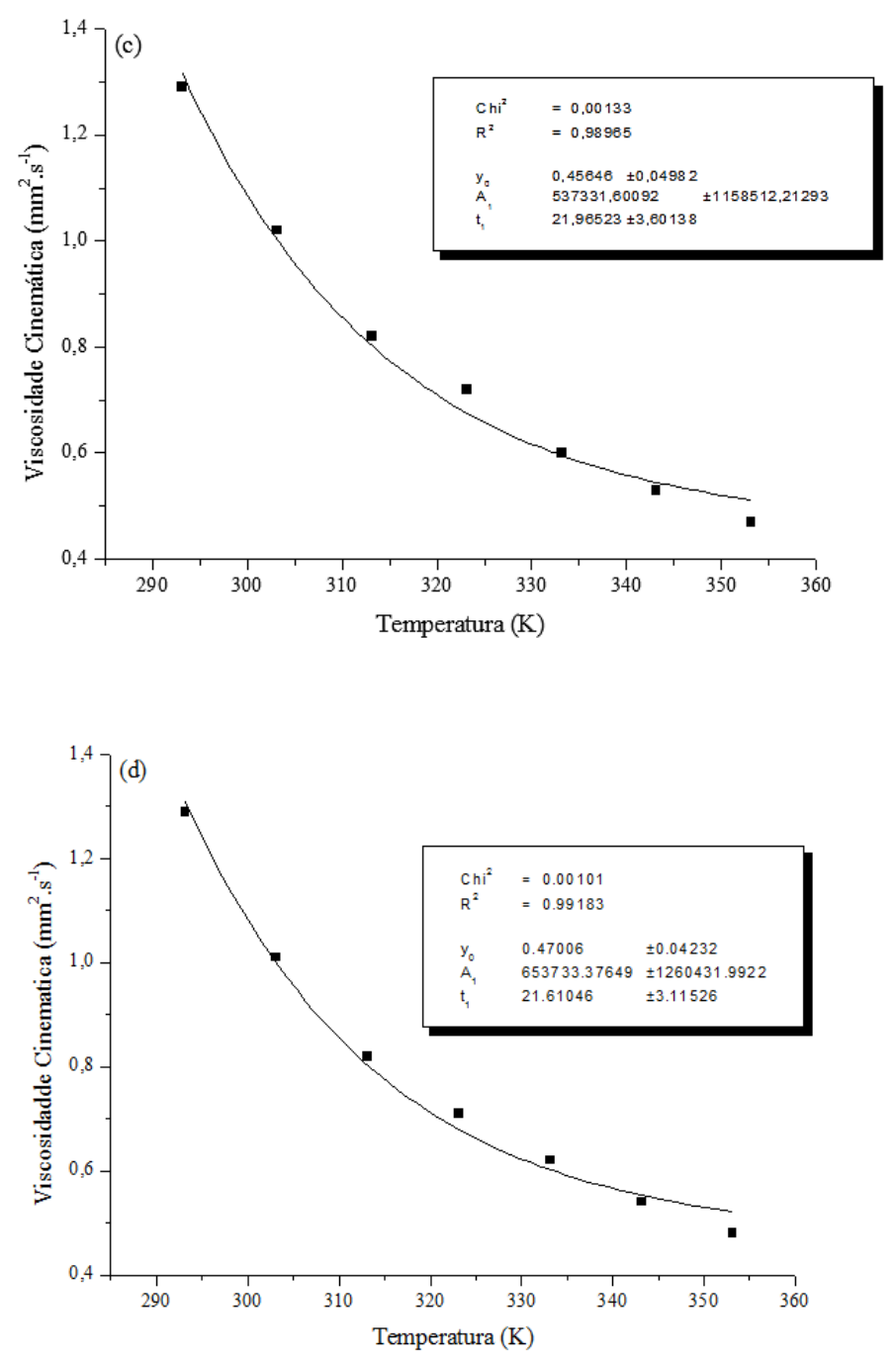

Figura 5. Curvas dos dados experimentais de viscosidade em função da temperatura para as soluções de pectinas. a: Pectina cítrica industrial; b: $P$. edulis; c: $P$. alata; $\mathrm{d}: P$. quadrangularis

O teste de ajuste de qui-quadrado $\left(\mathrm{Chi}^{2}\right)$ é usado para avaliar se há uma relação entre os dados esperados e observados. Quando as frequências observadas e esperadas apresentam uma diferença pequena, o teste $\mathrm{Chi}^{2}$ adquire valores próximos a 0 (zero). ${ }^{46}$ Os valores de $\mathrm{Chi}^{2}$ encontrados para o ajuste dos dados das viscosidades das soluções de pectina encontram-se entre 0,0010 e 0,0014, tendo os seus coeficientes de determinação valores entre 0,98 e 0,99 para todas as amostras, demonstrando assim um bom ajuste para as curvas de viscosidades.
Para a obtenção dos dados viscosimétricos foram utilizadas as Equações 4 a 7. As equações de Huggins $(H)$, Kraemer (K), Martin (M) e Schulz-Blaschke (SB) foram aplicadas no método de determinação da viscosidade intrínseca por extrapolação gráfica. A Figura 6 ilustra as retas obtidas por meio da equação de Huggins. Os valores escolhidos para os intervalos de concentração garantem que o trabalho foi realizado em regime newtoniano. Tal fato pode ser comprovado pela relação linear observada na Figura 3 para todas as soluções. 


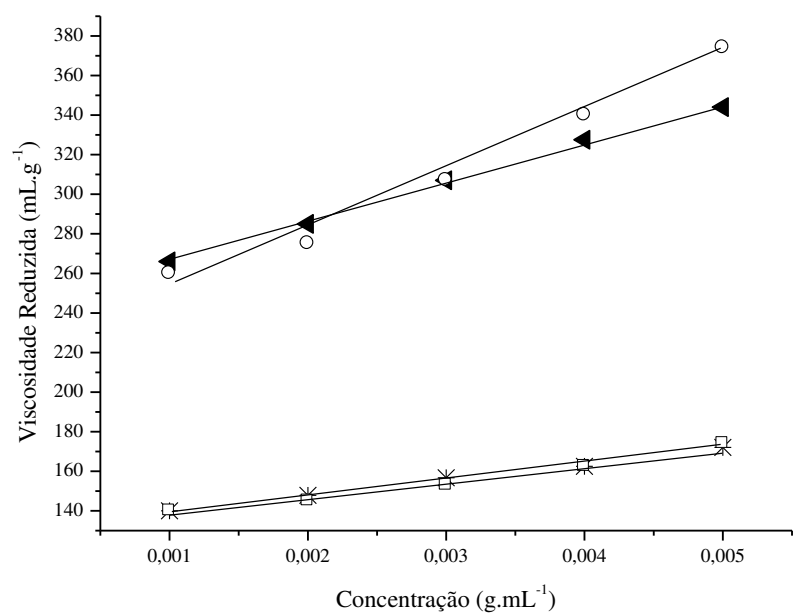

Figura 6. Viscosidade intrínseca das soluções de pectina. o Pectina Cítrica industrial $\left(R^{2}: 0,997\right)$; 4 P. edulis $\left(R^{2}: 0,984\right) ; \square P$. alata $\left(R^{2}: 0,996\right) ; * P$. quadrangularis $\left(R^{2}: 0,979\right)$

A Tabela 2 apresenta os valores de viscosidade intrínseca relacionados a todas as equações e a Tabela 3 mostra as constantes viscosimétricas de Huggins, Kraermer, Martin e Schulz-Blascke obtidas para todas as soluções de pectina. A constante de Huggins, $k_{h}$, fornece informações sobre as interações polímero-solvente. Quanto menor for o seu valor, melhor deverá ser o solvente, ou seja, mais solvatado deverá estar o polímero. Resultados experimentais indicam que valores menores que 0,5 são obtidos para soluções poliméricas diluídas em um bom solvente. O volume hidrodinâmico é o volume ocupado por um novelo (molécula) polimérica isolado em uma solução ${ }^{47}$. A constante de Kraemer $\left(k_{k}\right)$ depende da razão entre o volume hidrodinâmico ocupado por duas moléculas de soluto (polímero) que interagem entre si e o volume hidrodinâmico ocupado por uma molécula isolada. ${ }^{28,35}$

Tabela 2. Valores de viscosidade intrínseca para todas as amostras de pectina a $25{ }^{\circ} \mathrm{C} \mathrm{em}$ solução tampão fosfato de sódio $0,01 \mathrm{M} \mathrm{e} \mathrm{pH} 7$

\begin{tabular}{|c|c|c|c|c|c|c|c|}
\hline \multicolumn{8}{|c|}{ Solução de Fosfato de Sódio 0,01 M, pH 7} \\
\hline \multicolumn{8}{|c|}{ Viscosidade Intrínseca $[\eta]\left(m L^{-1}{ }^{-1}\right)$} \\
\hline Amostras & $\mathrm{H}^{\mathrm{a}}$ & $K^{\mathrm{a}}$ & $M^{a}$ & $\mathrm{SB}^{\mathrm{a}}$ & $\mathrm{SB}^{\mathrm{b}}$ & $\mathrm{SC}^{\mathrm{C}}$ & $D C^{c}$ \\
\hline $\begin{array}{l}\text { Pectina cítrica } \\
\text { industrial }\end{array}$ & 246,35 & 240,16 & 245,03 & 253,92 & 240,00 & 239,89 & 455,89 \\
\hline P. edulis & 223,30 & 232,13 & 222,75 & 239,02 & 255,45 & 256,12 & 559,65 \\
\hline P. alata & 132,20 & 131,92 & 132,01 & 134,76 & 138,39 & 138,39 & 78,22 \\
\hline P. quadrangularis & 129,25 & 131,11 & 129,92 & 132,14 & 139,73 & 139,73 & 80,63 \\
\hline
\end{tabular}

(H: Huggins; K: Kraermer; M: Martin e SB: Schulz-Blascke; ${ }^{2}$ Calculados por extrapolação gráfica;

${ }^{b}$ Calculado através da determinação por um único ponto $\left(\mathrm{k}_{\mathrm{sb}}=0.28\right)(\mathrm{SB}$ : Schulz-Blascke);

${ }^{\circ}$ Calculados através da determinação por um único ponto (SC: Solomon-Ciuta e DC: Deb-

Chanterjee). 
Tabela 3. Constantes de Huggins, Kraemer, Martin e Schulz-Blascke para as soluções de pectinas em tampão fosfato de sódio $\mathrm{pH} 7$ a 0,01 M

\begin{tabular}{cccccc}
\hline Amostras & $\mathbf{K}_{\mathbf{h}}$ & $\mathbf{K}_{\mathbf{k}}$ & $\mathbf{K}_{\mathbf{m}}$ & $\mathbf{K}_{\mathbf{s b}}$ & $\mathbf{K}_{\mathbf{h}}+\mathbf{K}_{\mathbf{k}}$ \\
\hline Pectina cítrica industral & 0,3218 & $-0,1388$ & 0,2769 & 0,0014 & 0,1830 \\
P. edulis & 0,6044 & $-0,0789$ & 0,4652 & 0,0023 & 0,5255 \\
P. alata & 0,4554 & $-0,0897$ & 0,4009 & 0,0020 & 0,3657 \\
P. quadrangularis & 0,5357 & $-0,0068$ & 0,4497 & 0,0024 & 0,5425
\end{tabular}

$\mathrm{K}_{\mathrm{h}}$ : Constante de Huggins; $\mathrm{K}_{\mathrm{k}}$ : Constante de Kraemer; $\mathrm{K}_{\mathrm{m}}$ : Constante de Martin; $\mathrm{K}_{\mathrm{sb}}$ : Constante de Schulz-Blascke.

Quando o coeficiente de Kraemer é negativo significa que há uma boa solvatação da cadeia polimérica, ou seja, há maior interação polímero-solvente. ${ }^{28,35,48}$

A Tabela 3 mostra que as soluções de pectina (cítrica e $P$. alata) apresentaram valores de $K_{h}<0,5$ apesar de o valor obtido para $P$. edulis e P.quadrangularis seja maior que 0,5 , os valores negativos para a constante de Kraermer, $\mathrm{K}_{\mathrm{k}}$ indicam uma boa interação entre polímero e solvente. Já os valores de $\mathrm{k}_{\mathrm{sb}}$ obtidos para as amostras por extrapolação gráfica, por serem bem inferiores a 0,28 variando de 0,0014 a 0,0024, (Tabela 3), demonstram que o uso destes métodos por determinação por um único ponto, torna-se ineficiente para este sistema (polímero-solvente), tendo produzido valores de $[\eta]_{s b}$ muito distantes dos obtidos por extrapolação gráfica (Tabela 2).

É importante observar que das três espécies estudas juntamente com a pectina cítrica industrial somente a $P$. edulis e $P$. quadrangularis apresentaram valores de $\mathrm{k}_{\mathrm{h}}+$ $k_{k}=0,5$, que segundo a literatura é uma premissa para que as equações de SolomonCiuta e Deb-Chanterjee sejam aplicadas (Tabela 3). ${ }^{28}$

No entanto, tanto $[\eta]_{s c}$ quanto $[\eta]_{d c}$ apresentaram valores distantes de $[\eta]_{h},[\eta]_{k}$, $[\eta]_{\mathrm{m}}$ e $[\eta]_{\mathrm{sb}}$, que foram obtidas por extrapolação gráfica (Tabela 2), com diferenças percentuais altas. Portanto, a aplicação das equações SC (Equação 13) e DC (Equação 14), para as soluções de pectina, no respectivo solvente analisado, mostrou-se ineficaz.

A massa molecular média viscosimétrica $\left(\mathrm{M}_{\mathrm{v}}\right)$ da pectina em solução tampão fosfato de sódio foi calculada a partir da equação de Mark-Houwink-Sakurada, de acordo com as constantes $K=0,3$ e $\alpha=0,613$, fornecida pela literatura para este sistema polímerosolvente a $298,15 \mathrm{~K}^{34,49}$

A massa molecular da pectina pode ser variável de acordo com a origem vegetal, matéria-prima e condições de extração, o que torna a sua determinação um desafio devido aos problemas de heterogeneidade (composição química variada) e agregação (aproximação das cadeias poliméricas), além da usual larga distribuição das cadeias poliméricas de pectina. Comparativamente, os valores de massa molecular de pectina são, notadamente, menos elevados que os da celulose ou do amido. Os valores citados na literatura para fontes alternativas de pectina variam entre 20.000 a 360.000 g.mol ${ }^{-1}$ e as amostras comerciais entre 35.000 a 120.000 g. $\mathrm{mol}^{-1}$, segundo o tipo de pectina, de baixo ou alto teor do seu grau de esterificação. $^{45,50,51}$ A Tabela 4 mostra os valores das massas moleculares para todas as equações. 
Tabela 4. Valores de massa molecular média viscosimétrica em g.mol ${ }^{-1}$ para as pectinas de quatro fontes diferentes, determinadas a partir das viscosidades intrínseca de Huggins $\left(\mathrm{Mv}_{\mathrm{h}}\right)$, Kraermer $\left(\mathrm{Mv}_{\mathrm{k}}\right)$, Martin $\left(\mathrm{Mv}_{\mathrm{m}}\right)$, Schulz-Blascke $\left(\mathrm{MV}_{\mathrm{sb}}\right)$, Solomon-Ciuta $\left(\mathrm{Mv}_{\mathrm{sc}}\right)$ e Deb-Chanterjee $\left(\mathrm{Mv}_{\mathrm{DC}}\right)$

\begin{tabular}{cccccccc}
\hline Amostras & ${ }^{\mathrm{a}} \mathbf{M v}_{\mathbf{h}}$ & ${ }^{\mathrm{a}} \mathbf{M v}_{\mathbf{k}}$ & ${ }^{\mathrm{a}} \mathbf{M} \mathbf{v}_{\mathbf{m}}$ & ${ }^{\mathrm{a}} \mathbf{M v}_{\mathbf{s b}}$ & ${ }^{\mathbf{b}} \mathbf{M v}_{\mathbf{s b}}$ & ${ }^{\mathbf{b}} \mathbf{M v}_{\mathbf{s c}}$ & ${ }^{\mathbf{b}} \mathbf{M v}_{\mathbf{D c}}$ \\
\hline Pectina cítrica industrial & 56,800 & 54,493 & 56,307 & 59,678 & 54,434 & 54,393 & 155,088 \\
P. edulis & 48,400 & 51,552 & 48,197 & 54,072 & 60,266 & 60,524 & 216,629 \\
P. alata & 20,578 & 20,506 & 20,529 & 21,231 & 22,172 & 22,172 & 8,741 \\
P. quadrangularis & 19,830 & 20,301 & 20,002 & 20,562 & 22,523 & 22,523 & 9,185 \\
\hline
\end{tabular}

${ }^{a}$ Calculados por extrapolação gráfica; ${ }^{b}$ Calculados através da determinação por um único ponto

A Tabela 5 apresenta as diferenças percentuais $(\Delta \%)$ calculadas para os valores de viscosidade intrínseca obtidos pelas equações de Kraemer, Martin, Schulz-
Blaschke, Solomon-Ciuta e Deb-Chanterjee, quando comparados com os valores produzidos pela equação de Huggins.

Tabela 5. Diferenças percentuais (\%) obtidas para valores de viscosidade intrínseca

\begin{tabular}{cccccccc}
\hline \multicolumn{8}{c}{ Diferenças percentuais para os valores de viscosidade intrínseca $(\Delta \%)$} \\
\hline \multicolumn{7}{c}{ Solução tampão fosfato de sódio $\mathrm{pH} \mathrm{7}$} \\
Emostras & $\mathrm{K}$ & $\mathrm{M}$ & $\mathrm{SB}$ & $\mathrm{SB}$ & $\mathrm{SC}$ & $\mathrm{DC}$ \\
Pectina cítrica industrial & $-2,56$ & $-0,53$ & 3,07 & $-2,57$ & $-2,62$ & 85,1 \\
\hline P. edulis & 3,95 & $-0,24$ & 7,03 & 14,4 & 14,7 & 150 \\
P. alata & $-0,21$ & $-0,14$ & 1,93 & 4,68 & 4,68 & $-40,8$ \\
P. quadragularis & 1,43 & 2,13 & 2,23 & 8,10 & 8,10 & $-37,6$ \\
\hline
\end{tabular}

Calculadas por extrapolação gráfica (equações de Kraemer (K), Martin (M) e Schulz-Blaschke (SB)) e medidas por um único ponto [equações de Schulz-Blaschke (SB), Solomon-Ciuta (SC) e

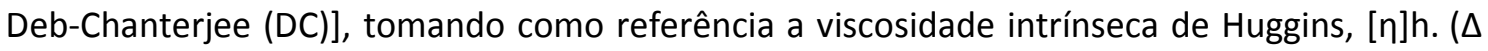
$\%)^{\mathrm{a}}=\left[100\left([\eta] /[\eta]_{\mathrm{h}}\right)\right]-100$.

O uso do método de determinação por um único ponto possibilita caracterizar soluções poliméricas de forma cada vez mais rápida. Em sua aplicação, seus dados são comparados com os resultados obtidos por extrapolação gráfica, método mais usado, a fim de estabelece se as condições dos sistemas poliméricos (polímero-solvente) são adequadas para o seu uso, sendo sua incerteza obtida por uma variação percentual que dirá se a margem de erro está segura para sua aplicação. ${ }^{28}$
Podemos observar que os valores obtidos pelos métodos de determinação por um único ponto apresentam as maiores variações mediante comparação com os valores obtidos por extrapolação gráfica. Ressaltando a equação de Deb-Chanterjee (DC), sendo esta a que apresentou maior ineficácia para o cálculo da viscosidade intrínseca das soluções de pectina. O método de extrapolação gráfica é o mais indicado para determinação da viscosidade intrínseca para este sistema polímero-solvente. 
As diferenças percentuais $(\Delta \%)^{\mathrm{b}}$ obtidas para os valores de massa molecular média viscosimétrica são mostradas na Tabela 6 .
Esses valores foram calculados levando-se em conta o valor de Mv, determinado pela equação de Huggins como referência. ${ }^{28,35}$

Tabela 6. Diferença percentual $(\Delta \%)$ obtida por valores de massa molecular viscosimétrica média. $(\Delta \%)^{\mathrm{b}}=\left[100\left(\mathrm{M}_{\mathrm{v}} / \mathrm{M}_{\mathrm{vk}}\right)\right]-100$

\begin{tabular}{ccccccc}
\hline \multicolumn{7}{c}{ Diferenças Percentuais $(\Delta \%)$} \\
\hline Amostras & $\mathrm{Mv}_{\mathrm{k}}$ & $\mathrm{Mv}_{\mathrm{m}}$ & $\mathrm{Mv}_{\mathrm{sb}}$ & $\mathrm{Mv}_{\mathrm{sb}}$ & $\mathrm{Mv}_{\mathrm{sc}}$ & $\mathrm{Mv}_{\mathrm{DC}}$ \\
Pectina cítrica industrial & 4,23 & 0,87 & $-4,82$ & 4,34 & 4,42 & $-63,3$ \\
P. edulis & $-6,11$ & 0,42 & $-10,5$ & $-19,9$ & $-20,0$ & $-77,6$ \\
P. alata & 0,35 & 0,23 & $-3,07$ & $-7,18$ & $-7,19$ & 135,4 \\
P. quadragularis & $-2,32$ & $-0,85$ & $-3,55$ & $-11,9$ & $-11,9$ & 115,8 \\
\hline
\end{tabular}

Kraermer $\left(\mathrm{Mv}_{\mathrm{k}}\right)$, Martin $\left(\mathrm{Mv}_{\mathrm{m}}\right)$, Schulz-Blascke $\left(\mathrm{MV}_{\mathrm{sb}}\right)$, Solomon-Ciuta $\left(\mathrm{Mv}_{\mathrm{sc}}\right)$ e Deb-Chanterjee $\left(\mathrm{Mv}_{\mathrm{DC}}\right)$.

Os valores de $(\Delta \%)$ mostraram uma tendência em sua diminuição para todas as amostras com o aumento da massa molecular média viscosimétrica. Os valores de massa moleculares médias obtidos por meio da determinação da viscosidade intrínseca por um único ponto apresentaram as maiores variações, não sendo útil para a determinação das massas moleculares da pectina.

As dimensões das cadeias poliméricas, (Tabela 7), foram obtidas, a partir dos dados viscosimétricos referentes à equação de Huggins, por meio da equação de Flory. ${ }^{28,52}$

Tabela 7. Distância média quadrática $\left(\mathrm{h}^{2}\right)$

\begin{tabular}{cc}
\hline Amostra & $\left(\mathbf{( h}^{\mathbf{2}} \mathbf{)}\right.$ \\
\hline Pectina cítrica industrial & $5,44 \times 10^{-22} \mathrm{~cm}$ \\
P. edulis & $3,70 \times 10^{-22} \mathrm{~cm}$ \\
P. alata & $4,66 \times 10^{-23} \mathrm{~cm}$ \\
$P$. quadrangularis & $4,26 \times 10^{-23} \mathrm{~cm}$ \\
\hline
\end{tabular}

Através da equação de Flory foi possível ter um indicativo das dimensões das cadeias poliméricas de pectina. Pode-se notar uma relação decrescente (Cítrica $>$ P.edulis $>P$. alata $>$ P.quadrangularis). Tal relação pode ser constada pelos valores de viscosidade intrínseca, (Tabela 2), e massa molecular viscosimétrica média, (Tabela 4), obtidas pela equação de Huggins e Mark HouwinkSakurada. Isto implica que para maiores valores de viscosidade, tende-se a ter cadeias poliméricas mais estendidas e com uma alta massa molecular.

\section{Conclusão}

As soluções de pectina das espécies de maracujás $(P$. edulis, $P$. alata, $P$. 
quadrangularis) e cítrica industrial se caracterizam como fluído newtoniano. Quando observados seus comportamentos em função do aumento da temperatura pode-se observar uma redução da viscosidade cinemática para todas as soluções. Este resultado foi comprovado pelas relações lineares obtidas através da concentração crítica de orvelap, confirmando que as soluções de pectina em soluções diluídas apresentam tal comportamento.

A regressão exponencial das curvas de viscosidade cinemática revelou ótimos valores de $\mathrm{Chi}^{2}$ e coeficientes de determinação $(0,9)$, demonstrando uma ótima interação entre polímero e solvente. Estas afirmações podem ser observadas através das constantes de Huggins para as soluções de pectina (Cítrica e P.alata), uma vez que seus valores são 0,3218 e 0,4554, apesar de as soluções de $P$.edulis $(0,6044)$ e $P$.quadrangularis $(0,5357)$ apresentarem valores de $\mathrm{K}_{\mathrm{H}}$ maiores que 0,5 ; a constante de Kraermer, por sua vez, apresentou valores negativos para todas as soluções, evidenciando uma boa solvatação para as cadeias poliméricas de pectina no solvente.

$O$ método de determinação por um único ponto, utilizando as equações de SchulzBlaschke, Solomon-Ciuta e Deb-Chanterjee, mostrou-se ineficaz para a determinação das viscosidades intrínsecas das quatro espécies de pectina estudadas em solução tampão fosfato de sódio $\mathrm{pH} \mathrm{7,} \mathrm{pois} \mathrm{os} \mathrm{desvios}$ apresentaram valores muito altos, não sendo eficaz para a determinação da viscosidade intrínseca para este sistema. A equação de Schulz-Blaschke quando aplicada na determinação por um único ponto apresentou resultados muitos superiores quando comparados com os obtidos por extrapolação gráfica.

A distância média quadrática extremo-aextremo das cadeias poliméricas das pectinas foi determinada pela equação de Flory, evidenciando uma relação decrescente do tamanho das cadeias poliméricas de pectina com sua viscosidade e massa molecular média viscosimétrica (pectina cítrica industrial $5,44 \times 10^{-22}>P$. edulis $3,70 \times 10^{-22}>$
P. alata $4,66 \times 10^{-23}>P$. quadrangulares 4,26 $\left.\times 10^{-23}\right)$.

As pectinas das três espécies de maracujá estudadas apresentaram comportamento reológico similar ao da pectina cítrica industrial, o que mostra a sua qualidade. Com os conhecimentos obtidos no campo reológico pode-se agregar a estas espécies um grande valor tecnológico no mercado industrial de alimentos, possibilitando o uso de novas fontes usuais para extração de pectina.

\section{Agradecimentos}

Ao CNPq e a Universidade Federal de Roraima.

\section{Referências Bibliográficas}

${ }^{1}$ Faleiro, F. G.; Juqueira, N. T. V.; Braga, M. F. Maracujá Germoplasma e Melhoramento Genético. Embrapa Cerrados. Planaltina-DF, 2005.

${ }^{2}$ Figueiredo L. P.; Valente, W. A.; Dias, M. V.; Borges, S. V.; Pereira, P. A. P.; Pereira, A. G. T.; Clemente, P. R. Efeito da Adição de Suco de Maracujá e Tempo de Cozimento sobre a Qualidade de Doces do Albedo de Maracujá em Calda. Ciência e Tecnologia de Alimentos 2009, 4, 840. [CrossRef]

${ }^{3}$ Sitio do Instituto Brasileiro de Geográfica e Estatística. Disponível em: <http://www.ibge.gov.br/>. Acesso em: 16 março 2016.

${ }^{4}$ Santos, J. L. V., Resende, E. D.; Martins, D. R.; Gravina, G. A.; Cenci, S. A.; Maldonado, J. F. M. Determination of the Harvest Point of Different Passion Fruit Cultivars. Revista Brasileira de Engenharia Agrícula Ambiental 2013, 7, 750. [CrossRef]

${ }^{5}$ Coelho, A. A.; Oliveira, E. M. S.; Resende, E. D.; Thiébaut, J. T. L. Dimensionamento Amostral para a Caracterização da Qualidade Póscolheita do Maracujá-Amarelo. Revista Ceres 2011, 1, 23. [Link] 
${ }^{6}$ Oliveira, L. C.; Santos, J. A. B.; Narain, N.; Fontes, A. S.; Campos, R. S. S.; Souza, T. L. Caracterização e Extração de Compostos Voláteis de Resíduos do Processamento de Maracujá (Passiflora edulis Sims f. flavicarpa degener). Ciência Rural 2012, 12, 2280. [CrossRef]

${ }^{7}$ Bernacci, L. C.; Meletti, L. M. M.; SoaresScott, M. D. Maracujá-doce: o Autor, a Obra e a Data da Publicação de Passiflora alata (Passifloraceae). Revista Brasileira de Fruticultura 2003, 2, 355. [CrossRef]

${ }^{8}$ Rodrigues, V. E. G.; Carvalho, D. A. Levantamento Etnobotânico de Plantas Medicinais do Domínio Cerrado na Região do Alto Rio Grande - Minas Gerais. Ciência Agrotécnica 2001, 1, 102. [Link]

${ }^{9}$ Silva, D. B. da.; Silva, J. A. da.; Junqueira, N. T. V.; Andrade, L. R. M. Frutos do cerrado. Embrapa, Informação Tecnológica. BrasíliaDF, 2001. [Link]

${ }^{10}$ Pontes, M. A. N.; De Holanda, L. F. F.; Uria, H. F.; Barroso, M.A.T. Estudo dos Subprodutos do Maracujá (Passiflora edulis f. flavicarpa deg.): III - Obtenção do Óleo e da Torta. Ceppa 1989, 1, 23. [CrossRef]

${ }^{11}$ Ferrari, R. A.; Colussi, F.; Ayub, R. A. Caracterização de Subprodutos da Industrialização do MaracujáAproveitamento das Sementes. Revista Brasileira de Fruticultura 2004, 1, 101. [CrossRef]

${ }^{12}$ Zeraik, M. L.; Perreira, C. A. M.; Zuin, V. G.; Yariwake, J. H. Maracujá, um Alimento Funcional?. Revista Brasileira de Farmacologia 2010, 20, 459. [CrossRef]

13 Jarvis, M. C., Forsyht, W.; Duncan, H. J. A Survey of the Pectic Content of Nonlignified Monocot Cell Walls. Plant Physiology 1988, 2, 309. [CrossRef]

${ }^{14}$ Shkodina, O. G.; Zeltser, O. A.; Selivanov, N. Y.; Ignatov, V. V. Enzymic Extraction of Pectin Preparations from Pumpkin. Food Hydrocolloids 1998, 3, 313. [CrossRef]

${ }^{15}$ Brandão, E. M.; Andrade, C. T. Influência de Fatores Estruturais no Processo de Gelificação de Pectinas de Alto Grau de Metoxilação. Polímeros 1999, 3, 38. [CrossRef]
${ }^{16}$ Paiva, E. P.; Lima, M. S.; Paixão, J. A. Pectina: Propriedades Químicas e Importância sobre a Estrutura da Parede Celular de Frutos Durante o Processo de Maturação. Revista Ibero-americana de Polímeros 2009, 4, 196. [Link]

17 Thakur, B. R.; Singh, R. K.; Handa, A. K. Chemistry and uses of Pectin - A review. Food Science and Nutrition 1997, 1, 47. [CrossRef]

${ }^{18}$ Willats, W. G. T.; Knox, J. P.; Mikkelsen, J. D. Pectin: New Insights into an Old Polymer are Starting to Gel. Trends in Food Science and Technology 2006, 3, 97. [CrossRef]

${ }^{19}$ Fietz, V. R.; Salgado, J. M. Efeito da Pectina e da Celulose nos Níveis Séricos de Colesterol e Triglicerídeos em Ratos Hiperlipidêmicos. Ciência e Tecnologia de Alimentos 1999, 3, 318. [CrossRef]

${ }^{20}$ Piedade, J.; Canniatti-Brazaca, S. G. Comparação entre o Efeito do Resíduo do Abacaxizeiro (Caules e Folhas) e da Pectina Cítrica de Alta Metoxilação no Nível de Colesterol Sanguíneo em Ratos. Ciência $e$ Tecnologia de Alimentos 2003, 2, 149. [CrossRef]

${ }^{21}$ Terpstra, A. H.; Lapre, J. A.; De Vries, H. T.; Beynen, A.C. Dietary Pectin With High Viscosity Lowers Plasma and Liver Cholesterol Concentration and Plasma Cholesteryl Ester Transfer Protein Activity in Hamsters. The Journal of Nutrition 1998, 11, 1944. [Link]

${ }^{22}$ May, C. D. Industrial Pectins: Sources, Production and Applications. Carbohydrate Polymers 1990, 1, 79. [CrossRef]

${ }^{23}$ Gnanasambandam, G.; Practor, A. Preparation of Soy Hull Pectin. Food Chemistry 1999, 4, 461. [CrossRef]

${ }^{24}$ Sitio da Revista-fi. Disponível em: < http://www.revista-

fi.com/materias/380.pdf>. Acesso em: 18 março 2016.

${ }^{25}$ Mello, I. L.; Coutinho. F. M. B.; Delpech, M. C.; Albino, F. F. M.; Santos, S. M. Viscometric Study of High-cis Polybutadiene in Toluene Solution. Polímeros 2006, 1, 194. [CrossRef]

${ }^{26}$ Sitio associação Brasileira de Poliméros. Disponível em: <http://www.reologiadobrasil.com.br/reologi a.html>. Acesso em: 11 março 2016. 
${ }^{27}$ Horta, A.; Sáiz, E.; Barrales-Rienda, J. M.; Gómez, P. A. G. Direct Estimation of the True Mark-Houwink-Sakurada Parameters from Gel Permeation Chromatography, Intrinsic Viscosity and one Average of Molecular Weight. Polymer 1986, 1, 139. [CrossRef]

${ }^{28}$ Lucas, E.F., Soares, B.G. \& Monteiro, E. Caracterização de Polímeros: Determinação do Peso Molecular e Análise Térmica. 1ạ. ed., e-papers. Rio de Janeiro, 2011.

${ }^{29}$ Kliemann, E. Dissertação de Mestrado. Universidade Federal de Santa Catariana, 2006. [Link]

${ }^{30}$ Pinheiro, E. R. Dissertação de Mestrado. Universidade Federal de Santa Catariana, 2007. [Link]

${ }^{31}$ Sitio do IAL-Instituto Adolfo Lutz. Normas analíticas. Métodos químicos e físicos para análise de alimentos. São Paulo, 2009. Disponível em: <http://www.ial.sp.gov.br>. Acesso em: 04 julho 2015.

${ }^{32}$ Canteri, M. H. G.; Scheer, A. P.; Ginies, C., Renard, C. M. G. C.; Wosiacki, G. Importance of Thermal Treatment on the Rind Passion Fruit to Pectin Extraction. Revista Brasileira de Tecnologia Agroindustrial 2010, 1, 109. [CrossRef]

${ }^{33}$ Oliveira, R. C.; Rossi, R. M.; Barros, S. T. D. Estudo Reológico da Polpa de Morango (Fragaria vesca) em Diferentes Temperaturas. Acta Scientiarum 2012, 3, 283. [CrossRef]

${ }^{34}$ Haminiuk, C. W. I.; Sierakowsik, M. R.; Izidoro, D. R.; Maciel, G. M.; Scheer, A. P.; Masson, M. L. Comportamento Reológico de Sistemas Pécticos de Polpas de Frutas Vermelhas. Ciência e Tecnóloga de Alimentos 2009, 1, 225. [CrossRef]

${ }^{35}$ Delpech, M. C.; Coutinho, F. M. B.; Habibe, M. E. S. Viscometry Study of Ethylene-Cyclic Olefin Copolymers. Polymer Testing 2002, 4, 411. [CrossRef]

${ }^{36}$ Solomon, O. F.; Ciuta, I. Z. Détermination de la Viscosité Intrinsèque de Solutions de Polymères par une Simple Détermination de la Viscosité. Journal of Applied Polymer Science 1962, 24, 683. [CrossRef]

${ }^{37}$ Deb, P. C.; Chantterjee, S. R. Unperturbed Dimension of Polymer Molecules from Viscosity Measurements. Indian journal of Applied Chemistry 1968, 1, 49. [CrossRef]
${ }^{38}$ Moreira, J. C., Silva, I. F., Wang, S. H., \& Balogh, D. T. Determinação das Constantes K e $\alpha$ da Equação de Mark-Houwink de Poli(pacetóxiestireno). Polímeros 2004, 2, 80. [CrossRef]

${ }^{39}$ Delpech, M. C.; Coutinho, F. M. B.; Habibe, M. E. S. Viscometry Study of Ethylene-Cyclic Olefin Copolymers. Polymer Testing 2002, 4, 411. [CrossRef]

${ }^{40}$ Glass, J. E.; Shulz, D. N.; Zukoski, C. F. Polymers as Rheology Modifiers An Overview. American Chemical Society 1991, 1, 2. [CrossRef]

${ }^{41}$ Junior, P. I. F.; Almeida, J. P. S.; Passos, S. R.; Oliveira, P. J.; Rumjanek, N.G.; Xavier, G.R. Produção e Comportamento Reológico de Exopolissacarídeos Sintetizados por Rizóbios Isolados de Guandu. Pesquisa agropecuária Brasileira 2010, 12, 1465. [Link]

${ }^{42}$ Sadicoff, B. L.; Brandão, E. M.; Lucas, E. F.; Amorim, M. C. V. Copolimerização Micelar de Poli(Acrilamida-g-Óxido de Propileno): Avaliação Reológica e Caracterização de suas Soluções. Polímeros 2001, 2, 41. [CrossRef]

${ }^{43}$ Valeri, D.; Meirelles, A. J. A. Viscosities of Fatty Acids, Triglycerides, and Their Binary Mixtures. Journal of American Oil Chemists Society 1997, 10, 1221. [Link]

${ }^{44}$ Ferreira, E. E.; Brandão, P. R. G.; Klein, B. Reologia de Suspensões Minerais: Uma Revisão. Revista Escola de Minas 2005, 1, 83. [Link]

${ }^{45}$ Canteri, M. H. G.; Scheer, A. P.; Ginies, C.; Renard, C. M. G. C.; Wosiacki, G. Pectina: da Matéria-Prima ao Produto Final. Polímeros 2012, 2, 149. [CrossRef]

${ }^{46}$ Farber L. Estatística Aplicada. Pearson Education. 4a. ed., São Paulo, 2010.

${ }^{47}$ Craciun, G.; Ighigeanu, D.; Manaila, E.; Stelescu, M. D. Synthesis and Characterization of Poly(Acrylamide-CoAcrylic Acid) Flocculant Obtained by Electron Beam Irradiation. Materials Research 2015, 5,984. [CrossRef]

${ }^{48}$ Silva, G. M.; Ferreira, I. L. M.; Delpech, M. C.; Costa, M. A. S. Estudo Viscosimétrico de Copolímeros à Base de 1,3-Butadieno e 1Octeno em Tulueno e Hexano. Polímeros 2013, 6, 758. [CrossRef]

${ }^{49}$ Arslan, N. Extraction of Pectin from SugarBeet Pulp and Intrinsic Viscosity-Molecular 
Weight Relationship of Pectin Solutions. Journal of Food Science and Technology 1995, 5, 381. [Link]

${ }^{50}$ Voragen, G. J., Pilnik, W., Thibault, J. F., Axelos, M. A. V., \& Renard, C. M. G. C. Pectins, in: Food Polysaccharides and their Applications. Marcel Dekker Inc. New York, 1991.
${ }^{51}$ Sakai, T.; Sakamoto, T.; Hallaert, J.; Vandamme, E. Pectin, Pectinase, and Protopectinase: Production, Properties, and Applications. Advances in Applied Microbiology 1993, 39, 213. [CrossRef]

52 Bianchi, O.; Repenning, G. B.; Mauler, R. S.; Oliveira, R. V. B.; Canto, L. B. Caracterização Viscosimétrica de Nanocompósitos Híbridos PS/POSS. Polímeros 2012, 2, 125. [CrossRef] 